\title{
ON A VARIATIONAL PROBLEM HAVING A THIRD ORDER DIFFERENTIAL EQUATION AS A NECESSARY CONDITION FOR AN EXTREMUM
}

\section{PHILIP COOPERMAN}

The object of the following note is to give an example of a variational problem in which the Euler-Lagrange equations are equivalent to a third-order differential equation. This example is not an artificial one, but arises from the physical problem of determining the electric potential in a gas through which a space-charge limited current flows. The appropriate differential equations are:

$$
\begin{aligned}
\nabla^{2} u & =-4 \pi \rho, \\
\nabla \cdot j & =0, \\
j & =-k \rho \nabla u,
\end{aligned}
$$

where

$$
\begin{aligned}
u & =\text { potential } \\
j & =\text { current density vector, } \\
\rho & =\text { space-charge density } \\
k & =\text { mobility of charge carrier }=\text { a given function, } \\
\nabla & =\text { vector operator }=(\partial / \partial x, \partial / \partial y, \partial / \partial z) \\
\nabla^{2} & =\text { Laplacian. }
\end{aligned}
$$

Elimination of $j$ from this system leads to the system of equations consisting of equation (1) and

$$
\boldsymbol{\nabla} \cdot[k \rho \nabla u]=0 .
$$

Similarly, it is possible to eliminate $\rho$ from (1) and (4) resulting in the single, nonlinear equation of third order

$$
\boldsymbol{\nabla} \cdot\left[k \nabla^{2} u \nabla u\right]=0 .
$$

It is clear that (5) is equivalent to (1) and (4) which in turn is equivalent to the original system.

Let us now consider the problem of determining an unknown function, $\rho$ such that the integrals

$$
E=\iiint\left[\frac{1}{2}(\nabla u)^{2}-4 \pi \rho u\right] d x d y d z
$$

and

Received by the editors July 9, 1953. 


$$
P=\iiint k \rho(\nabla u)^{2} d x d y d z
$$

are simultaneously extremized with respect to variations in $u$. From a physical viewpoint, this amounts to extremizing the energy stored, and the power dissipated in the region under consideration.

The Euler-Lagrange equations for this problem are equations (1) and (4) which, as shown above, are equivalent to equation (5). Thus, a third-order differential equation, namely equation (5), is a necessary condition for an extremum in this problem.

Research Corporation, Bound Brook, N. J.

\section{ON BERNSTEIN'S SELF-DUAL SET OF POSTULATES FOR BOOLEAN ALGEBRAS}

RICHARD MONTAGUE AND JAN TARSKI

In 1916 Professor B. A. Bernstein published an elegant, self-dual set of postulates for Boolean algebras. ${ }^{1}$ It was asserted at that time that the postulates were independent, but Mr. Robert Russell of the University of California discovered that the independence proofs for the commutative laws were erroneous. ${ }^{2}$ It has remained an open question whether each of these laws actually is independent of the other laws. The answer, as this note will establish, is negative.

We quote Bernstein's postulates, omitting those concerned with closure under the fundamental operations:
P1. $x+y=y+x$.
P3. $x+y \cdot z=(x+y) \cdot(x+z)$.
P2. $x \cdot y=y \cdot x$.
P4. $x \cdot(y+z)=x \cdot y+x \cdot z$.

P5. For every $x$, there is a $c$ such that, for every $u, u+x \cdot c=u$ and $u \cdot(x+c)=u$.

P6. There are $x$ and $y$ such that $x \neq y$.

We shall derive $\mathrm{P} 1$ from $\mathrm{P} 2-\mathrm{P} 5$.

Given $x$, let $c$ be an element such that, for every $u, u+x \cdot c=u$ and $u \cdot(x+c)=u$ (cf. P5).

Received by the editors June 1, 1953.

${ }^{1} \mathrm{~B}$. A. Bernstein, $A$ simplification of the Whitehead-Huntington set of postulates for Boolean algebras, Bull. Amer. Math. Soc. vol. 22 (1916) p. 458.

2 This error was acknowledged by Professor Bernstein in his article, A dual-symmetric definition of Boolean algebra free from postulated special elements, Scripta Mathematica vol. 16 (1950) p. 157. 